\title{
EL DIOS CHAAHK EN EL NOMBRE DE LOS GOBERNANTES MAYAS
}

\author{
Ana García Barrios \\ Universidad Complutense de Madrid
}

El trabajo de onomástica real que aquí se presenta nos ofrece información valiosa sobre la importancia que Chaahk tuvo entre los gobernantes del Clásico, además de que arroja nuevos datos sobre este dios. ${ }^{1}$

La epigrafía y la iconografía son disciplinas inseparables a la hora de elaborar un estudio sobre onomástica como éste. Afortunadamente en los últimos 20 años la epigrafía ha sufrido un avance espectacular y gracias a los investigadores en esta materia podemos conocer mejor la lectura de los nombres personales de gobernantes y otros personajes de la sociedad maya del período Clásico. Es fundamental empezar a denominarlos por sus verdaderos nombres. A pesar de que el camino del desciframiento a veces se hace demasiado largo, son ya muchos a los que podemos dar una lectura correcta.

Aquí se aportarán algunos de los nombres de gobernantes que en su estructura nominal incluían al dios Chaahk, esperando sirva como punto de partida a un futuro estudio que complemente el mapa antroponímico de toda el área maya durante el período Clásico.

Son diversas las cuestiones que quedan por estudiar sobre los nombres de los gobernantes mayas. Permanecen abiertas preguntas como ¿por qué se elegían determinados nombres? ¿Existe algún modelo patronímico que indique la relación de parentesco? ¿Solamente los gobernantes y su corte llevaban nombres de deidades?, ¿Existen apelativos vinculados a áreas concretas? Algunas de estas cuestiones se abordarán a lo largo de esta exposición.

En los últimos años, los trabajos de Carrasco (1964), Houston y Stuart (1996), Bricker (2002), o Grube (2002) han sacado a la luz nueva información sobre nombres personales, que ha servido para el entendimiento de su estructura y el acercamiento a patrones de parentesco en las líneas dinásticas.

Los nombres propios se forman a través de frases nominales que expresan una cualidad o característica del dios Chaahk, y en ocasiones se ven apoyados por otros nombres o sobrenombres, pudiendo incluir varias cláusulas nominales. Estas denominaciones son oraciones completas formadas por un sujeto:

\footnotetext{
${ }^{1}$ Este artículo forma parte de una investigación más amplia cuyo tema central es el dios Chaahk. Uno de los objetivos más importantes es conocer los apelativos con los que se denominó a Chaahk en la época prehispánica y cuáles de estos nombres han sobrevivido hasta nuestros días.
} 
Chaahk, y el predicado puede incluir un verbo u otros sustantivos. El sujeto ocupa siempre la última posición de la frase nominal (Grube, 2002).

Los reyes mayas formaron el eje de unión entre el pueblo y los dioses. La conexión con estos últimos debió realizarse de diversas maneras y es posible que el nombre fuese una forma de adquirir las poderosas cualidades del dios, pudiendo elegir identificarse con unos aspectos más que con otros.

Se desconocen los motivos por los que los reyes del Clásico prefirieron sólo algunos de los dioses del panteón para crear sus nombres propios. Curiosamente los más mencionados son K'awil, Yoopat y Chaahk, generalmente prefijados por el locativo chan, "Cielo", lo cual nos indica que eligen el aspecto celeste de los dioses y que la acción está ocurriendo en el cielo.

Sucede que estos tres dioses pueden compartir los mismos calificativos y oraciones nominales variando simplemente el sujeto, este es el caso del nombre de K'ahk' Tiliw Chan Chaahk, de la ciudad de Naranjo y K'ahk' Tiliw Chan Yoopat, de la ciudad de Quiriguá.

Estas tres deidades tienen una vinculación estrecha con los fenómenos que ocurren en el cielo, lo cual no implica que haya una división clara de atributos y actividades celestes y, aunque sea difícil de entender por nuestros arraigados conceptos occidentales, es posible que las tres interviniesen en este espacio y lo que en él ocurría. Todas comparten algunos distintivos como el hacha, objeto que se emplea para abrir, entre otros elementos, el cielo.

Chaahk, al igual que otros dioses como K'awiil, aparece actuando en los tres planos del mundo: el supramundo (representado por el cielo), el plano terrenal y el inframundo, siendo este último en donde posiblemente ocurre la gran mayoría de las escenas mitológicas de Chaahk — representadas en los vasos cerámicos tipo códice-. Sin embargo, los gobernantes prefirieron para sus apelativos el aspecto celestial del dios, a través del cual se relacionan con él y en el que se resaltan, de manera gráfica y descriptiva, las actitudes y los poderes del dios, mismos que el soberano posiblemente comenzaba a compartir desde el momento en que lo elegía como nombre personal.

Chaahk es por tanto un dios vinculado a la atmósfera y a lo que allí sucede. Lo primero que debe aclararse para saber qué implica el nombre de Chaahk, es su etimología, y para ello me he basado en un estudio linguístico elaborado en el año 2000 por Lacadena y Wichmann, sobre el desarrollo del término Chaahk, donde sostienen que su significado inicial es "Rayo". Proponen que chaahk es una palabra de procedencia cholana, cuya raíz proviene del vocablo protomaya kahwuq o kahwoq, que evolucionó hasta tomar la forma de chaahk durante el período Clásico. En la actualidad los pueblos de lengua cholana siguen empleando el término chajc para referirse al rayo.

A pesar de que se registra la presencia iconográfica de Chaahk desde el Preclásico Tardío, como se observa en la Estela 1 de Izapa, todo indica que el complejo linguístico e iconográfico que conocemos de Chaahk tiene su origen en la zona cholana de las Tierras Bajas del Sur, durante el período Clásico maya, y en 
un momento posterior la lengua yucateca lo tomó prestado de esta zona. Por estudios linguiísticos se sabe que esta región del norte fue bilingüe, escribiéndose textos tanto en cholano como en yucatecano, ${ }^{2}$ incluyendo los modismos del sur, lo cual se advierte en el nombre del gobernante de Uxmal K'ahk'Pul[aj] Chan Chaahk, "Chaahk ahumea en el cielo", cuya estructura y lengua son claramente cholanas.

Si partimos de esta idea, Chaahk en el período Clásico fue principalmente el dios del Rayo, y esto se ve apoyado por las frases nominales a las que aparece asociado. Fue también un dios vinculado a la lluvia, al trueno, al relámpago y a todos los aspectos atmosféricos de las tormentas eléctricas, tan comunes en estas regiones, pero en especial al rayo.

Autores como Taube (1992) o Grube (2002) relacionan a Chaahk sobre todo con las nubes y el fuego celeste, mientras que al dios K'awiil lo asocian con el relámpago y el trueno, atributos de los que con mayor asiduidad también participaba Chaahk. Por ahora no tenemos un desarrollo etimológico del nombre de K'awiil que pueda expresar por sí mismo su significado, y tampoco podemos acotar en qué planos actuaban cada uno de ellos individualmente, aunque queda claro que K'awiil es un dios de fuego, vinculado al poder de los gobernantes $\mathrm{y}$, por las representaciones iconográficas y escritas que nos han llegado, tan antiguo como Chaahk.

Los dioses durante el Clásico fueron denominados mediante apelativos que marcaban o resaltaban sus características atributivas, pudiendo aludir a cualquiera de ellas independientemente. Estos apelativos son los mismos que eligen muchos de los reyes mayas para sus nombres personales, escogiendo, como ya he mencionado, principalmente dioses que habitaban el espacio celeste. Son muy poco comunes las nominaciones que implican vínculos con la muerte o el inframundo, aunque es cierto que existen, como el famoso gobernante de Toniná llamado B’ak Nal Chaahk, "Chaahk el del sitio del hueso". Salvo estas raras excepciones, los gobernantes se identificaron con dioses que habitaban el cielo.

El motivo por el que preferían dioses celestes parece claro a primera vista, pues es fácil pensar según nuestra estructura occidental, que en el cielo habita el bien y que las acciones asociadas a él tienden a ser favorables.

Con la lectura detallada de estos nombres se aprecia que la intención última de los gobernantes es trasmitir el poder y el alcance de la grandeza del dios. Poder que a veces se veía traducido en rayo, relámpago o en cualquier otra acción, violenta o no, asociada al espacio celeste. Por tanto, se puede intuir que eligieron estos nombres por ser descriptivos y representativos por sí mismos; pues sin lugar a dudas la ferocidad de las tormentas debieron resultar insignias muy convincentes que resaltaban la majestad y el poder de la realeza.

\footnotetext{
${ }^{2}$ Yucatecano es la forma de referirse a la lengua hablada y escrita en la época prehispánica, para diferenciarla del yucateco actual.
} 
Durante los siglos VII, VIII y Ix, coincidiendo con el esplendor del Clásico Tardío, Chaahk fue uno de los dioses más mencionados en las cláusulas nominales de los gobernantes, pero estos nombres no sólo en este período fueron empleados, las estructuras nominales se mantienen en los siglos sucesivos, sobreviviendo incluso a la conquista española.

Treinta y tres gobernantes, ${ }^{3}$ de todos los que reinaron en el período Clásico, eligieron cláusulas nominales de Chaahk como nombre propio; aclaremos que éstos son los nombres propios con los que se les menciona en el momento de entronización o cuando ya están ejerciendo su reinado; no olvidemos que existen pruebas que demuestran que en ocasiones se efectuaba un cambio del nombre de infancia al tener acceso al poder (Grube, 2002); esto, que muy pocas veces aparece reflejado en los textos, da lugar a que sólo conozcamos al gobernante por el nuevo nombre elegido al llegar al trono.

Sobre cómo se realizaba la elección del nombre no tenemos información fidedigna, pero en la vida de un gobernante nada estaba sujeto al azar, así que probablemente se basaban en datos marcados por augurios protectores que debieron recogerse en los códices de ese entonces.

Los nombres registrados hasta ahora los encontramos asociados a dos regiones muy concretas:

La región del norte de Yucatán, donde sólo aparecen seis menciones en cuatro ciudades, y las Tierras Bajas del Sur, en las que vivieron 26 gobernantes, ubicados, por un lado, en la región petenera (Tikal, Naranjo, Machaquila) y por otro, en la franja del Usumacinta (Pomona, La Mar).

En la región intermedia, la formada por las ciudades de estilo Río Bec-Chenes —quizá por haber sido menos explorada—, únicamente se ha encontrado un texto con el nombre de un gobernante; está incompleto pero igualmente sitúa al dios en una acción celeste al quedar prefijado por el locativo chan, "Cielo".

Los nombres elegidos por dichos gobernantes fueron muy variados, en ellos encontramos diversos apelativos que nos muestran las cualidades y características del dios, además de aspectos físicos del mismo. Sin embargo, en este trabajo solamente se presentan algunas de las cláusulas nominales de Chaahk que incluyen aspectos o fenómenos atmosféricos, y se han dividido en:

- Los que aparecen asociados a términos pluviales.

- Los que incluyen términos de fuego y por tanto relacionados directamente con el rayo o el relámpago.

A pesar de que etimológicamente el nombre de Chaahk signifique "rayo", - y ésta es una de las principales razones para vincular su desarrollo linguiístico

\footnotetext{
${ }^{3}$ Estos son los gobernantes con apelativos de Chaahk que se han registrado hasta el momento. Entendiéndose que no es una investigación cerrada y que el campo de la arqueología no dejará de revelar nuevos nombres personales.
} 
a las Tierras Bajas del Sur-Chaahk se asocia también, como ya se ha comentado, a otros fenómenos atmosféricos: niebla, lluvia, trueno, relámpago, todas ellas cualidades especificas del dios, incluidas en su esencia.

Chaahk, por un lado, es fuego al desdoblarse en rayo o relámpago, y fue uno de los apelativos que más emplearon los gobernantes mayas. Por otro lado, es agua o lluvia, que por lo general y en especial en el norte, se entiende como principio generador de vida, aunque sabemos que Chaahk poseía también los aspectos negativos de la lluvia encarnados en diluvio, inundaciones e incluso huracanes.

Sin embargo, los gobernantes del Clásico no mostraron un interés especial por el aspecto pluvial de Chaahk, ${ }^{4}$ pues fueron muy pocos los que eligieron apelativos con términos como lluvia o nube. Esto parecería lógico en las Tierras Bajas del Sur, donde existe una importante red fluvial y un elevado índice de precipitaciones. Curiosamente es aquí donde se encuentran los dos únicos nombres de gobernantes asociados a los glifos que significan nube y niebla, mientras que en el norte de Yucatán, los gobernantes optaron por nombres de estilo "sureño", debido principalmente a las poderosas influencias y modismos procedentes de aquella región.

La única mención encontrada en Yucatán referente a Chaahk en su aspecto de lluvia es la de un alto dignatario de Chichén que su nombre era Yax Ha'al Chaahk, "Chaahk es la primera lluvia", apelativo que durante el Clásico fue muy empleado para referirse al dios y cuya pervivencia se atestigua en los períodos posteriores, el Posclásico y la Colonia, llegando hasta nuestros días.

\section{Nombres con connotaciones pluviales}

Los nombres que tienen connotaciones pluviales se encuentran escritos en varios textos y contextos:

En la pared de la cueva de Naj Tunich (figura 1) existe la referencia a un gobernante que debió realizar una peregrinación a este santuario, su nombre era Muyal Chaahk, "Chaahk es la nube", formado por dos sustantivos. Ésta es la composición mínima de una frase nominal.

Este mismo apelativo lo tenemos en la ciudad de Bonampak, asociado al dios

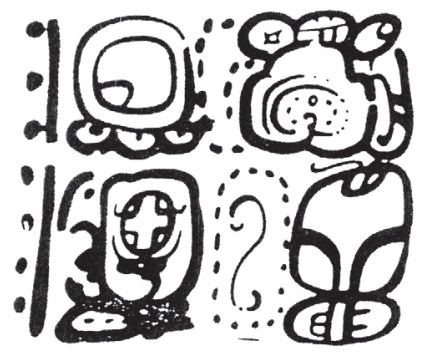

Figura 1. Muyal ChaAhk, "ChaAhk es la nUBE", Cueva de Naj Tunich Dibujo de Moisés Aguirre

\footnotetext{
${ }^{4}$ En el período Clásico se encuentran diversos apelativos con connotaciones relativas a la lluvia, asociados a contextos religiosos, pero son escasas las menciones en nombres de gobernantes.
} 
K'awiil, "K'awil es la nube del cielo". En este ejemplo va acompañado del locativo chan, lo que lleva a suponer que en Naj Tunich se pudo producir una abreviatura del nombre y que éste fuese Muyal Chan Chaahk, aunque el término donde ocurre la acción, chan, el cielo, no aparezca. Es lógico pensar que el sustantivo nube se encuentre asociado a cielo y que a pesar de que no se escriba se sobreentienda su ubicación, pues es el único lugar donde se pueden encontrar y de donde pueden proceder las nubes.

La segunda mención a apelativos pluviales la encontramos en el nombre del gobernante de la ciudad de Naranjo (figura 2), Yax Mayuy Chan Chaahk, "Chaahk es la primera niebla del cielo", que curiosamente es uno de los pocos encontrados en esta ciudad que no está asociado a glifos que indiquen fuego. En este caso la estructura es más compleja y se especifica qué tipo de niebla es: la primera, y donde se localiza la niebla: en el cielo.

Ahora bien, se repite la fórmula tan

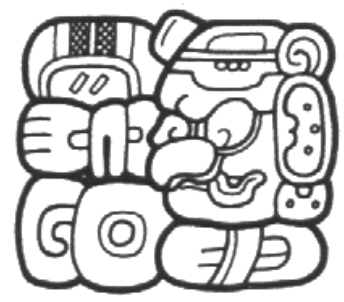

Figura 2. Yax Mayuy Chan ChaAhk, “Chaahk es la primera niebla del cielo", de la ciudad de Naranjo. Dibujo de N. Grube, 2002: 78 extendida para Chaahk en la que las acciones ocurren en el cielo, chan chaahk, y que comparte con otros gobernantes de esta misma ciudad y con otros dioses que también ocupan planos celestes.

\section{Nombres que incluyen términos de fuego}

Los apelativos de Chaahk relativos a fuego, quemar o arder, son los más comunes en el período Clásico, tanto en el norte como en el sur de las Tierras Bajas, debido en parte a las connotaciones que el fuego celeste tiene con el rayo.

La ciudad de Naranjo es un ejemplo claro de la veneración hacia este dios, donde tres de los cinco gobernantes que llevaron nombres de Chaahk lo asociaron a fenómenos que implican fuego, relampaguear, quemar y el término ukalaw (siguiendo a Grube, 2002), glifo que por el momento no tiene traducción, aunque la estructura de la oración indica que el signo kal igualmente debe ser un término de fuego.

Todos estos gobernantes emplearon la misma estructura variando únicamente la acción que realiza el dios y todos aparecen prefijados por el signo k'ahk', "fuego":

- K'ahk'Tiliw Chan Chaahk "Chaahk quema en el cielo" (figura 3)

- K'ahk' Yipiiy Chan Chaahk "Chaahk relampaguea en el cielo"

- K'ahk' Ukalaw Chan Chaahk "Chaahk (acción de quemar) en el cielo"

(figura 4) 


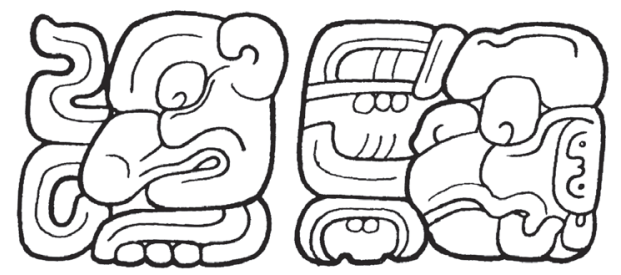

Figura 3. K'ahk' Tiliw Chan ChaAhK, “ChaAhk quema en el cielo", de la ciudad de Naranjo. Dibujo de N. Grube, 2002: 74

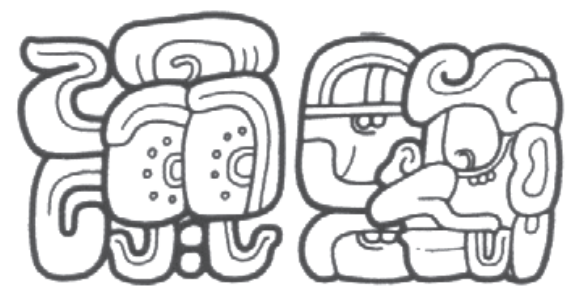

Figura 4. K'ahk' Ukalaw Chan ChaAhк, "Chaahk ? en el cielo", de la ciudad de Naranjo. Dibujo de N. Grube, 2002: 80

Estos tres ejemplos tienen en común el empleo de la fórmula antipasiva propuesta por Lacadena en el año 2000. En ellos no se ha traducido el signo k'ahk', debido a que seguimos la línea de pensamiento de algunos autores que opinan que k'ahk' puede ser un determinativo semántico, es decir, un signo que acompaña y que indica, en este caso, una acción relativa a fuego, pero que no tendría valor de lectura, tratándose por tanto de un signo silencioso (comunicación personal, A. Lacadena, 2004).

En la región Puuc se repite la estructura de los apelativos del sur, como se aprecia en el nombre del rey de Uxmal, K'ahk'P'ulaj Chan Chaahk, "Chaahk ahumea en el cielo" (figura 5). La localización de esta misma composición cholana en ciudades tan distantes como Naranjo y Uxmal, hace pensar que efectivamente éstas pasaron a formar parte de la élite yucateca y que muchos de los nombres encontrados en esta región tienen su origen en las Tierras Bajas del Sur, probablemente debido a la poderosa influencia y control que el sur ejercía sobre el norte. 


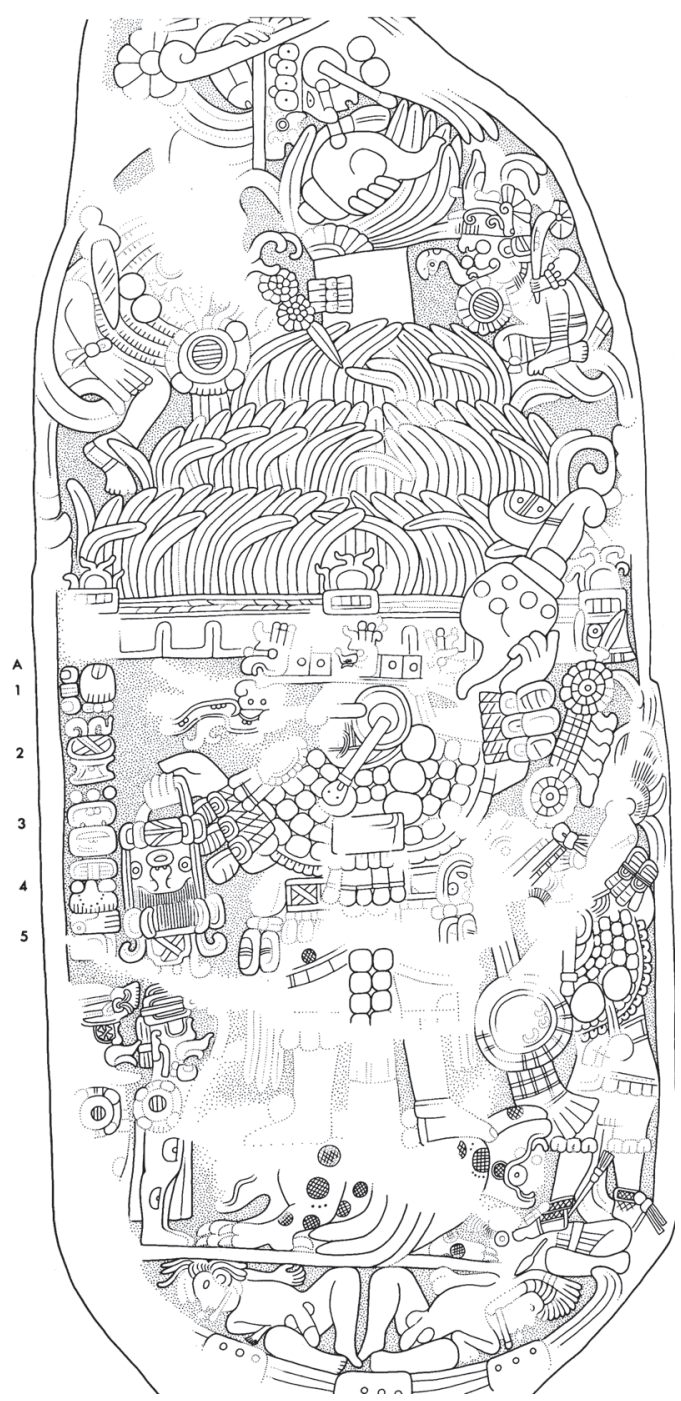

Figura 5. Estela 14, Uxmal. Dibujo de I. Graham, CHI

Existen otros nombres de gobernantes asociados a acciones de quemar o arder, a pesar de no registrarse en la oración el término k'ahk', "fuego". Se trata de aquellos que incluyen el sustantivo taj, "antorcha".

En el Clásico Temprano vivieron dos gobernantes que también usaron el término taj, antorcha:

- K'inich Tajal Chaahk, “Chaahk es la antorcha de K'inich”, gobernante de la ciudad de Naranjo, cuya única mención la encontramos en el plato K5458 (fi- 
gura 6). El otro gobernante fue Tajal Chaahk, "Chaahk Antorchado", de la ciudad de Tikal. Ambos nombres se refieren a la acción de Chaahk como generador del rayo, fenómeno atmosférico simbolizado mediante la antorcha.

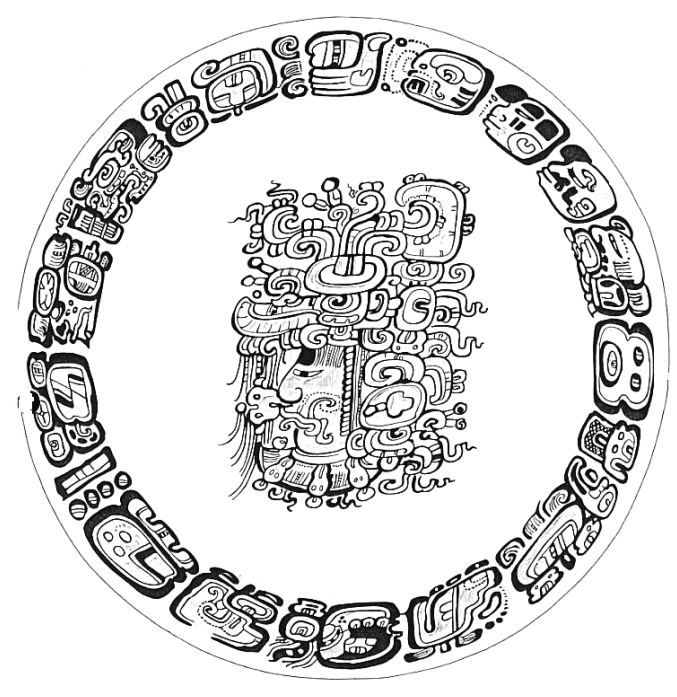

Figura 6. K'inich Tajal ChaAhK, de la ciudad de Naranjo Photograph copyright Justin Kerr, File no. K3067

Hay que resaltar que existe constancia de nombres de gobernantes en los que no se involucra la presencia nominal de dioses, este es el caso del gobernante de la ciudad de Calakmul, Tajoom u K'ab' K'ahk', "La Antorcha es el brazo de fuego". En estos apelativos se muestra una relación de procedencia del rayo, por eso es fácil pensar que estos atributos son siempre de deidades y que aunque no se mencione explícitamente el nombre del dios, se sobreentiende el hecho, acción o dios al que se está aludiendo. Asimismo, la traducción de estos apelativos ofrecen significados similares a otros donde sí aparece el nombre del dios actuando como sujeto, esto permite sugerir que en ocasiones los reyes haciendo gala del dominio de la lengua eligiesen como nombre personal únicamente la parte descriptiva del dios, evitando incluir el sujeto en la cláusula nominal, que en este caso sería Chaahk.

Es cierto que en las representaciones iconográficas la antorcha es un elemento muy recurrente, y en los códices encontramos distintos seres de carácter sobrenatural portándolas, como guacamayos (Dresde, 40B) y perros (Dresde, 36A) pero Chaahk es el único dios que aparece con ellas, representando su aspecto de rayo. 
Por tanto, se pretende dejar abierta la posibilidad de encontrarnos ante apelativos de dioses a pesar de que en ellos no se incluya al sujeto de la acción, en este caso Chaahk, probablemente porque se esté refiriendo a una característica tan peculiar del dios que no fue necesario incluir el nombre del mismo.

Muchos de estos apelativos de dioses permanecen vivos en el tiempo y no son exclusivos del período Clásico, pues se reconocen en los textos que nos han llegado del Posclásico, como vemos en el Códice de Dresde.

Tok ti Ka'an Chaahk, "Chaahk quema en el cielo". El significado de este apelativo tiene un origen Clásico, es el nombre del famoso gobernante de la ciudad de Naranjo, K'ahk' Tiliw Chan Chaak. La versión yucateca incluye el término tok, "quemar", que sustituye al verbo cholano til al compartir el mismo significado (figura 7).

En los textos mayas de la Colonia, como el Chilam Balam de Chumayel, se encuentran frases que incluyen nuevamente el locativo chan y que hacen referencia, entre otros, a los tipos de lluvias relacionados con los puntos cardinales, asociaciones muy comunes en este tipo de textos:

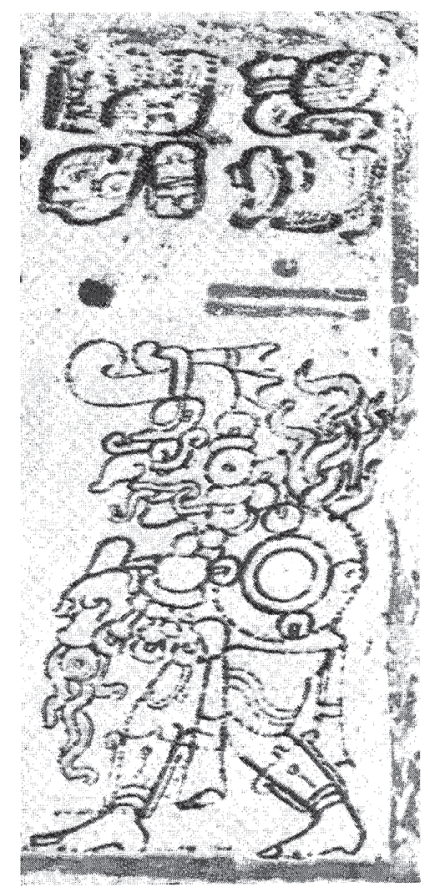

Thul Caan Chac Il

Boh ol Caan Chac Il

Ch'uh Um Caan Chac Il

Figura 7. TOK TI K'AN CHAAHK, "ChaAhK Quema en el CIelo" Códice Dresde, 36A.

Estos apelativos llegan hasta la actualidad pudiéndose reconocer aún en las oraciones y rezos de los pueblos mayas del norte de Yucatán, como los que recoge Villa Rojas en sus estudios sobre los mayas de Quintana Roo:

$\begin{array}{ll}\text { Lelem Ka'an Chac } & \text { "Chaahk del cielo relampagueante" } \\ \text { Bolon Ka'an Chac } & \text { "Chaahk del noveno cielo" } \\ \text { Mizeen Ka'an Chac } & \text { "Chaahk del cielo limpio" }\end{array}$

Igual que los apelativos de dioses sobreviven en la actualidad, tenemos datos que demuestran que también sobrevivieron como parte de los nombres personales, conservándose la estructura nominal del Clásico al menos hasta el siglo xviII. 
Con la llegada de los españoles aparentemente las tradiciones se rompen y los nombres se pierden, pero esto no es del todo cierto. Los frailes encargados de evangelizar a los indígenas comenzaron por darles un nombre cristiano, sin comprender que aquellos nombres que usaban no eran como los suyos, y suponiendo que estaban formados por simples nombres y apellidos.

Sin averiguar a qué hacían referencia cada una de esas cláusulas nominales, optaron por cambiar el nombre profano por uno del santoral cristiano y así, gracias a ese desconocimiento, pueden encontrarse dos siglos después de la Colonia supervivencias de los nombres que emplearon los mayas del Clásico.

En el pueblo de Dolores del Lacandón, el 25 de junio de 1697, se hace una "Memoria de los indios casados, solteros y viudos", se trata de un censo donde se describen todos los nombres de las personas que habitaban el pueblo. Encontramos entre otros, a Julián Chanchac, Baltasar BolonChac y Sebastian Bolonchac, posiblemente los últimos apelativos de Chaahk encontrados hasta el momento.

En esta primera memoria de 1697 (A.G.G., A1.11-2033-94, f.53-54, tomado de Vos, (1988: 266) y (A.G.I., Guat. 344, 3, f.60v-62) son muy escasas las menciones a Chaahk, apenas hemos encontrado cinco nombres que incluían al dios Chaahk. Cuando en 1712 se elabora la segunda memoria en este mismo pueblo de Dolores, sólo se registró un apelativo de Chaahk, que casualmente no coincidía con ninguno de los del censo anterior. Formaba parte del apellido de Martina Izchac, que significa: "Camote de Chac". Los apellidos del primer censo no aparecen porque en ese momento ya se había acabado con la población original del Sakbalam, repoblando el sitio con gente procedente de otras regiones del área maya, los que en la actualidad forman parte del grupo lacandón.

La escasa presencia de apelativos de Chaahk en estos censos indica que fueron nombres destinados a personas concretas, quizá sólo los gobernadores y su corte fueron los únicos que llevaron cláusulas nominales alusivas a dioses. En ese caso, Lestaríamos ante personajes de alto rango, pudiéndonos encontrar ante un posible ajaw todavía en el año 1697 ?

\section{Consideraciones finales}

La información global que se puede extraer de los nombres de gobernantes, es mucha y nos habla de acciones y actitudes concretas de los dioses. En el caso de Chaahk, sitúa al dios como un dios poderoso y con fuerza, capaz de crear tanto el bien como el mal. Los datos obtenidos nos proporcionan rasgos claros de los aspectos específicos elegidos por los gobernantes, con los que sin lugar a duda se identificaban. Estos soberanos optaron por incluir en sus nombres escenas celestes, fáciles de interpretar por aquellos que conocían su nombre, concretamente el pueblo maya y sus aliados o sometidos. Otros tipos de nombres son aquellos que hacen referencia a algún aspecto físico de Chaahk y por último, aquellos que están formados por adjetivos calificativos que indican cualidades del dios. 
Los nombres mayas se formaban partiendo de varias cláusulas nominales, y por los datos obtenidos de las crónicas coloniales, parte de estos nombres podrían hacer alusión a nombres de linaje, calpullis, barrios o subdivisiones territoriales que ubicarían el origen del portador (Carrasco Pizana, 1964: 327).

El hecho de que la mayoría incluya escenas celestes no implica que Chaahk sea exclusivamente un dios celeste, pues sus representaciones iconográficas demuestran que Chaahk actúa tanto en el Cielo como en el Inframundo.

Durante mucho tiempo se ha pensado que Chaahk era una deidad perteneciente a un momento tardío de la cultura maya y que su escenario estaría ubicado en las Tierras Bajas del Norte. Los abundantes mascarones de estilo PSUC, ${ }^{5}$ la sequía y la ausencia de ríos en esta región, además de las menciones del dios encontradas en códices y textos coloniales, favorecieron este pensamiento. Sin embargo, el estudio detallado de los nombres de Chaahk vincula su origen linguiístico y onomástico a la región de las Tierras Bajas del Sur, durante el desarrollo del período Clásico, de donde se tomaron los modelos y patrones que emplearon los gobernantes de la región yucateca.

La lectura e interpretación de estos apelativos nos permitirán conocer mejor la personalidad y características de Chaahk, pudiendo llegar a entender hasta donde alcanzaba su dominio, pues los nombres personales de los dioses son una fuente importantísima de información tanto para interpretaciones iconográficas como religiosas y mitológicas.

\section{Agradecimientos}

En primer lugar quisiera agradecer a Mario Humberto Ruz la confianza depositada en mí. Igualmente estoy en deuda con mi maestro y amigo personal Alfonso Lacadena, quien en todo momento me ha asesorado en el campo de la epigrafía. Por último, mi agradecimiento a Fernando Gómez por los comentarios y sugerencias así como su apoyo informático.

\section{BIBLIOGRAFÍA}

Berlin, $\mathrm{H}$.

1958 "El glifo emblema en las inscripciones mayas", Journal de la Société des Americanistes, vol XLVII: 11-119. Francia: Société des Americanistes, Musée de l'Homme.

\footnotetext{
${ }^{5}$ Durante muchas décadas se ha creído sin duda alguna, que estos mascarones eran la representación iconográfica de Chaahk, pero desde hace unos años permanece abierto el debate sobre a quién y qué representan estos mascarones tan característicos del norte de Yucatán.
} 
Brady, J. E.

2001 "Los oscuros secretos de los mayas: la exploración arqueológica de las cuevas”, Los mayas, N. Grube (ed.), pp. 297-310. Colonia, Alemania: Könemann Verlagsgesellschaft.

Bricker, Victoria

1990 A Morpheme Concordance of the Book of Chilam Balam of Chumayel. Nueva Orleáns: Tulane University (Institute Publication, 59).

2002 "Evidencia de doble descendencia en las inscripciones de Yaxchilán y Piedras Negras", La organización social entre los mayas, Memoria de la Tercera Mesa de Palenque, (I): 125-146. México: Conaculta, INAH.

Carrasco Pizana, P.

1964 "Los nombres de persona en la Guatemala Antigua", Estudios de Cultura Maya, 4: 323-334. México: UNAM, IIFL, Centro de Estudios Mayas.

Coe, Michael D.

1977 The Maya Scribe and His World. Nueva York: The Grolier Club New York.

1995 El desciframiento de los glifos mayas. México: FCE.

De Vos, Jan

1988 La paz de Dios y del Rey. La conquista de la Selva Lacandona (1525-1821). México: Secretaria de Educación y Cultura de Chiapas - FCE.

Grube, Nicolai

2002 "Onomástica en los gobernantes mayas", La organización social entre los mayas, Memoria de la Tercera Mesa de Palenque, (II): 323-353. México: Conaculta, INAH.

Houston, S. y David Stuart

1996 "Of gods, glyphs and kings: divinity and rulership among the Classic Maya", Antiquity, 70: 289-312, June 1996. Cambridge: Cambridge University Press.

Kerr, John

1989 The Maya Vase Book: A Corpus of Rollout Photographs of Maya Vases, B. Kerr y J. Kerr (eds.). Nueva York: Kerr Associates, 5 vols.

Lacadena García-Gallo, A.

2000 "Antipassive constructions in the Maya gliphic texts", Written Languages and Literacy, vol (II)-1: 155-180. Filadelfia: E. John Benjamin Publishing Company.

2004 "On the reading of two glyphic apellatives of the rain god", Acta Mesoamericana, vol. XIV. Continuity and Change, Maya Religious Practices in Temporal Perspective, pp. 87-98. Bonn: Verlag Anton Saurwein. 
Lacadena García-Gallo, A. y S. Wichmann

2002 "The distribution of Lowland Maya languages in the Classic period", Memoria de la Tercera Mesa Redonda de Palenque, vol. II, pp. 277-319, Vera Tiesler Blos, Rafael Cobos y Merle Greene Robertson (coords.). México: Conaculta, INAH.

Lacadena García-Gallo, A. y S. Wichmann

en prensa "On the representation of the glottal stop in Maya writing", The Linguistics of Maya Writing, Soren Wichmann (ed.). Salt Lake City: The University of Utah Press.

Lowe, Garret, Thomas Lee y E. Martínez

1982 Izapa: An Introduction to the Ruins and Monuments. Provo, Utah: Brigham Young University (Paper of the New World Archaeological Foundation, 31).

Martin, Simon y Nicolai Grube

2002 Crónica de los reyes y reinas mayas. Barcelona: Crítica.

Martínez Marín, Carlos

1972 "Santuarios y peregrinaciones en el México prehispánico", Religión en Mesoamérica, Jaime Litvak King y Noemí Castillo Tejeros (eds.), pp. 161-176. México: XII Mesa Redonda de la Sociedad Mexicana de Antropología.

Miller, Mary y Karl, Taube

1997 An Illustrated Dictionary of the Gods and Symbols of Ancient Mexico and Maya. Londres: Thames and Hudson.

Proskouriakoff, Tatiana

1960 "Historical implications of a pattern of dates at Piedras Negras, Guatemala", American Antiquity, 25 (4): 454-475. Salt Lake City: The Society for American Archaeology.

1963 "Historical data in the inscriptions of Yaxchilan (part I), Estudios de Cultura Maya, 3: 149-167. México: UNAM, IIFL, Centro de Estudios Mayas.

Rivera Dorado, M.

1986 Chilam Balam de Chumayel, M. Rivera (ed.). Madrid: Historia 16.

2001 La ciudad maya un escenario sagrado. Madrid: Complutense.

Ruz, Mario H.

1990 Los legítimos hombres. México: UNAM, IIFL, Centro de Estuidos Mayas, vols. I y II.

Schele, Linda y M. Miller

1986 The Blood of Kings: Dynasty and Ritual in Maya Art. Nueva York: George BraziIler Inc. en asociación con el Museo de Arte Kimbell, Fort Worth. 
Shellhas, P.

1978 Representation of Deities of the Maya Manuscripts, 2a. ed., revisión y traducción de Selma Wesselhoeft y A. M. Parker. Nueva York: Harvard University y Kraus Reprint Co. (Paper of the Peabody Museum of American Archaeology and Ethnology, Harvard University).

Taube, $\mathrm{K}$.

1992a The Major Gods of Ancient Yucatan. Washington D.C.: Dumbarton Oaks (Studies of Precolumbian Art \& Archaelogy, 32).

1992b The Temple of Quetzalcoatl and the Cult of Sacred War of Teotihuacan, RES, 21: 53-87. (hhtp://www.res-journal.org ).

2001a "Classic Maya gods", Maya: Divine Kings of the Rainforest, pp. 262-277, Nikolai Grube (ed.). Cologne: Könemann Verlagsgesellschaft .

2001b "Maya deities", Archaeology of Ancient Mexico and Central America: An Encyclopedia, pp. 431-433, Susan T. Evans y David L. Webster (eds.). Nueva York: Garland Publishing

Thompson, J. E.

1988 Un comentario al Códice de Dresde, libro de jeroglíficos. México: FCE. 